\title{
Identificación rápida de Lutzomyia longipalpis a partir de cinco descriptores del aparato genital masculino
}

\author{
Llano, E.G.; Maidana, H.R; Cabrera, W.R.; Báez, A.D.; Ledesma, R.M.
}

Servicio de Diagnóstico de Leishmaniosis, Hospital de Clínicas, Facultad de Ciencias Veterinarias, Universidad Nacional del Nordeste, Sargento Cabral 2139, Corrientes (3400), Argentina. Tel: 379-4425753.

E-mail: anato2@vet.unne.edu.ar.

\begin{abstract}
Resumen
Llano, E.G.; Maidana, H.R; Cabrera, W.R.; Báez, A.D.; Ledesma, R.M.: Identificación rápida de Lutzomyia longipalpis a partir de cinco descriptores del aparato genital masculino. Rev. vet. 24: 1, 21-24, 2013. El flebótomo Lutzomyia longipalpis es, hasta el momento, el vector comprobado de la leishmaniosis visceral canina. Capturas realizadas en Corrientes (Argentina) permitieron identificar a Lutzomyia neivai, vector de la leishmaniosis cutánea, así como a Lutzomyia migonei y Lutzomyia cortelezzii-sallesi, ambos considerados vectores secundarios de la leishmaniosis cutánea. Para el control epidemiológico resulta primordial la identificación del vector. Luego de la captura de los insectos, el paso más complicado es el reconocimiento de las distintas especies de Lutzomyia, para lo cual es necesario lograr el aclaramiento de sus estructuras y observarlo al microscopio prestando atención a los descriptores característicos y particulares de la especie. Existen aproximadamente 30 descriptores para identificar Lutzomyia longipalpis por la observación microscópica de su aparato genital masculino. El objetivo de este trabajo fue seleccionar cinco descriptores de fácil identificación, que en orden de importancia resultaron ser: setae diferenciadas del parámero, relación entre longitud del parámero y lóbulo lateral, forma y apariencia del coxito o basistilo, características y ubicación del mechón de setae del coxito y morfología de la bomba eyaculadora. La sencilla visualización de estos descriptores permite una rápida identificación taxonómica de la especie.
\end{abstract}

Palabras clave: Lutzomyia longipalpis, leishmaniosis, vector, identificación rápida.

\begin{abstract}
Llano, E.G.; Maidana, H.R; Cabrera, W.R.; Báez, A.D.; Ledesma, R.M.: Quick identification of Lutzomyia longipalpis considering five taxonomic key descriptors of the masculine genital apparatus. Rev. vet. 24: 1, 21-24, 2013. The phlebotomus Lutzomyia longipalpis is considered the vector of canine visceral leishmaniasis. Specimens captured in Corrientes (Argentina) allowed the identification of the species Leishmania neivai, vector of cutaneous leishmaniasis, and Leishmania migonei and Leishmania cortelezzii-sallesi, both considered secondary vectors of cutaneous leishmanisasis. After insects gathering, next step was the taxonomic identification of the different species of Lutzomyia by means of clearance of the anatomic structures and observation by light microscope, considering the characteristic descriptors for the species. There are approximately 30 descriptors used to identify L. longipalpis by microscopic observation of male genitalia. The aim of this study was to select five descriptors of easy identification, which were in order of importance: differentiated setae of paramere, relationship between length of paramere and lateral lobe, shape and presence of coxite or basistilo, characteristics and location of the setae tuft and morphology of the coxite ejaculatory pump. These descriptors allowed and easy visualization and rapid taxonomic identification for the species.
\end{abstract}

Key words: Lutzomyia longipalpis, leishmaniosis, vector, quick identification.

\section{INTRODUCCIÓN}

Los insectos agrupados en la subfamilia Phlebotominae (Diptera: Psychodidae) son hematófagos de

Recibido: 18 diciembre 2012 / Aceptado: 6 marzo 2013 importancia sanitaria. De las 700 especies existentes en el mundo, poco menos de 500 se han registrado en América y dentro de éstas, cerca de 30 actúan como vectores de Leishmania, Bartonella y varios arbovirus ${ }^{7,9,11}$. En Argentina se ha reportado un total de 28 especies, la mayoría de ellas pertenecientes al género 
Lutzomyia, y otras que conservan la denominación del genero Brumptomyia ${ }^{11}$. En la Ciudad de Corrientes (Argentina) las especies encontradas fueron Lutzomyia longipalpis (Lutz \& Neiva, 1912), vector de leishmaniosis visceral; Lutzomyia neivai (Pinto, 1926), vector de leishmaniosis cutánea; Lutzomyia migonei (França, 1920) y Lutzomyia cortelezzii-sallesi (Brèthes, 1923; Galvão \& Coutinho, 1939), vectores secundarios de leishmaniosis cutánea ${ }^{10}$.

La captura de Lutzomyia sp. se realiza mediante diversos métodos, según el objetivo perseguido en la investigación (tubos aspiradores, trampas atrayentes luminosas tipo CDC "Comunicable Disease Center", trampa Shannon con cebos o atrayentes luminosos y trampas de interferencia de vuelo adhesivas o pegajosas ${ }^{1,6,13}$. Las mini trampas CDC son artefactos dotados de una fuente luminosa, que atraen a los insectos merced a su fototropismo positivo. En esencia constan de una fuente lumínica, un tubo o envase plástico conectado a un ventilador (succiona al insecto) y una red o malla recolectora en la base (almacena los insectos succionados). Estas trampas pueden funcionar a batería o electricidad, por lo cual pueden instalarse a nivel domiciliario, peridomiciliario, periurbano o rural. Deben ser colocadas preferentemente en lugares con sombra vegetal, tierra húmeda, material en descomposición, en proximidad a sectores de vegetación densa y fuentes de ingesta sanguínea, sin interferencia de luces externas. Deben elegirse sitios donde hayan ocurrido casos recientes de leishmaniosis ${ }^{10}$.

En la región estudiada, la época más propicia para la captura incluye los meses de octubre a marzo. Las trampas deben colocarse a las $16-18 \mathrm{~h}$ y ser retiradas a las 07-09 h del día siguiente por un período de tres días consecutivos libres de lluvias, viento o disminución de temperatura. La altura de las trampas variará desde 1,20 a 2 m dependiendo del lugar donde se las ubique (dormideros de perros, gallineros, chiqueros, árboles). Deben evitarse posibles daños por la acción de perros, gatos, hormigas o arañas ${ }^{2}$. Tras la captura, los especimenes deben ser sacrificados sometiéndolos a la acción de éter, cloroformo o acetato de etilo en cámara cerrada, o bien por acción térmica a baja temperatura $\left(-20^{\circ} \mathrm{C}\right.$ durante $3 \mathrm{~h}$ ), así como por exposición a luz solar por igual período ${ }^{1}$.

Luego se realizará una inspección ocular en caja de Petri, sobre papel milimetrado a fin de seleccionar los especimenes que se asemejen al género buscado, teniendo en cuenta su tamaño y la posición de la cabeza con respecto al eje del cuerpo. Las maniobras se efectuarán con pinzas, ansas y lupa manual a efectos de no dañar el material ${ }^{2}$. El aclaramiento de los insectos se efectúa con la finalidad de poder observar con mayor claridad los órganos internos (cibario y espermateca en las hembras, bomba y filamentos eyaculadores en los machos). Para ello se pueden emplear soluciones de $\mathrm{NaOH}$ o $\mathrm{KOH}$ al 10-20\%, así como lactofenol (ácido láctico y fenol 1:1) o bien detergente diluido al 5\%. El tiempo de aclaramiento varía desde horas hasta un par de días ${ }^{8,12}$.
Para el examen microscópico el flebótomo es fijado a un portaobjeto mediante una gota de bálsamo de Canadá, diluida en fenol. Los especimenes pueden montarse enteros o seccionarse en cabeza (posición frontal), abdomen (posición dorsoventral o de perfil) y alas (totalmente extendidas) para apreciar con mayor detalle las características necesarias para su identificación ${ }^{3,8}$.

Para identificar microscópicamente ejemplares de Lutzomyia longipalpis de sexo macho, existen aproximadamente 30 descriptores del aparato genital, a saber: longitud total de la bomba genital, longitud del pistón, filamentos genitales (longitud, forma general del trayecto, trayecto de la porción terminal, forma de la porción terminal y estriación), lóbulo lateral (longitud, ancho y extremidad), parámero (número de las partes, setae diferenciadas, número de setae diferenciadas y distribución de las setae no diferenciadas del dorso), aedeago, coxito (longitud, ancho, número de hileras longitudinales, número de mechones de setae, posición de los mechones, número de setae de los mechones presentes, implantación de los mechones, tamaño de la zona de inserción del mechón del coxito, modo de agrupamiento de las setae del mechón y diferenciación de las setae del mechón), estilo (longitud, forma, setae subterminal, número de espinas y distribución de la inserción de cuatro espinas en el estilo) ${ }^{4-6,14}$

El objetivo de esta comunicación fue obtener y difundir una simplificación de la técnica de identificación de Lutzomyia longipalpis de sexo macho utilizando solamente cinco descriptores de sencilla visualización, que permitan una rápida ubicación taxonómica de la especie.

\section{MATERIAL Y MÉTODOS}

Utilizando una trampa de luz tipo CDC (Figura 1) suspendida a una altura de 1,20 m desde la reja de una ventana en el predio de la Facultad de Ciencias Veterinarias de la UNNE (Corrientes, Argentina), operando en el horario de 20:00 a 07:00 h, entre el 15 y 19 de

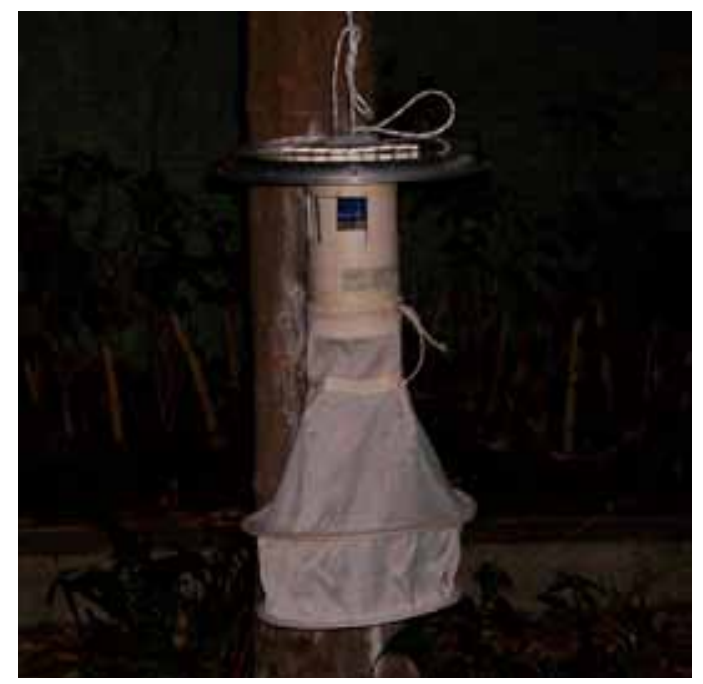

Figura 1. Trampa de luz utilizada para la captura del flebótomo. 
octubre de 2012 (tiempo de captura), 12 especimenes machos atrapados se identificaron como Lutzomyia longipalpis.

En el presente trabajo se investigaron únicamente ejemplares de sexo macho por ser el más atraído por las trampas de luz tipo CDC. Cabe aclarar que, en menor proporción, también fueron capturados ejemplares hembras como así también machos y hembras de otras especies.

A primera hora de la mañana se retiró la malla recolectora de la trampa, colocándola en un freezer durante 3-5 h para sacrificar a los insectos. Tras una primera observación macroscópica sobre papel milimetrado se descartaron los ejemplares cuyo tamaño o caracterís-

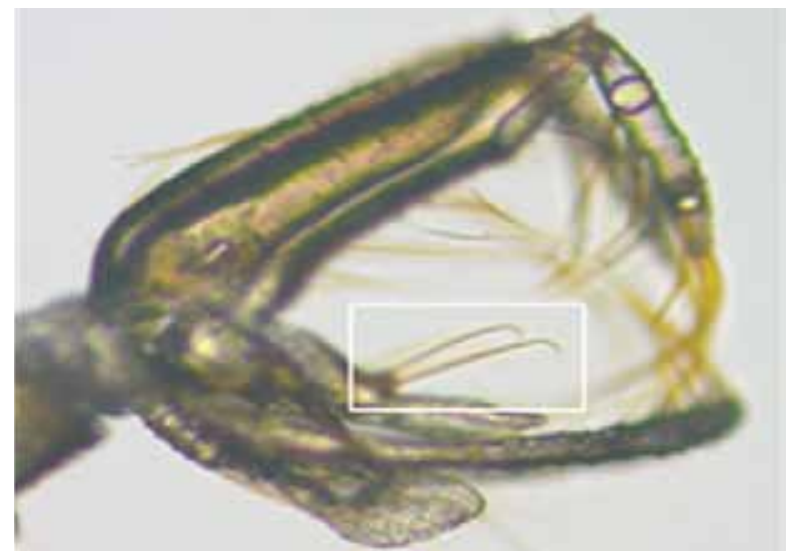

Figura 2. Setae diferenciadas del parámero.

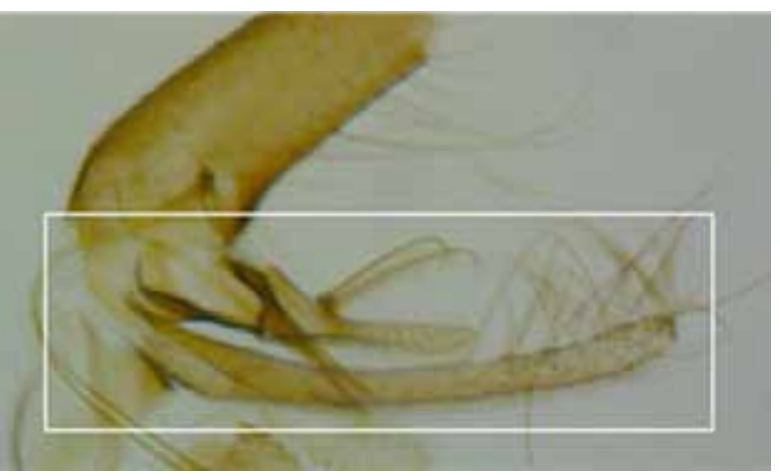

Figura 3. Relación entre longitud del parámero y lóbulo lateral 1:2.

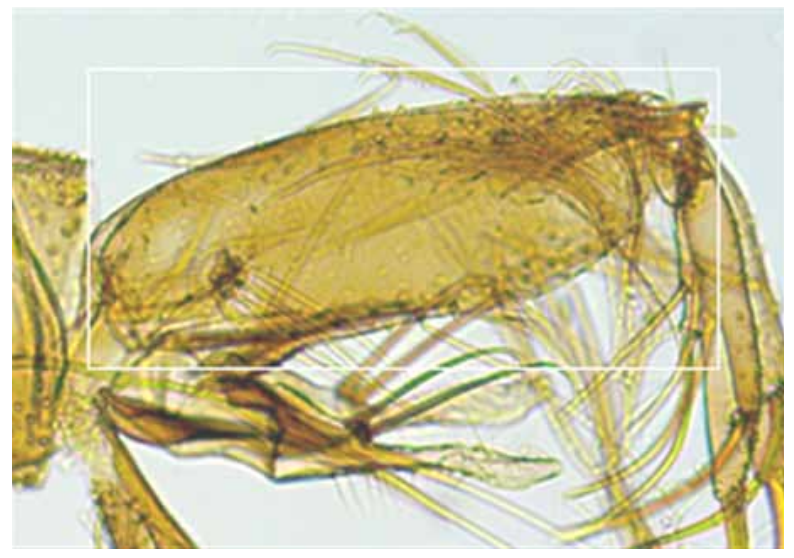

Figura 4. Coxito o basistilo, ancho y robusto. ticas no coincidieran con las del espécimen en estudio. Los restantes fueron fotografiados para disponer de un registro de cuerpo entero, tras lo cual fueron sumergidos en $\mathrm{KOH} 10 \%$ durante $24-48 \mathrm{~h}$ para su aclaramiento y para disminuir la pilosidad.

Luego fueron lavados durante una hora en etanol $70 \%$ para evitar la formación de cristales durante su observación al microscopio. El montaje en portaobjeto se concretó con aceite de inmersión y el examen se realizó a 4x, 10x, 40x y (colocando cubreobjeto) con objetivo de inmersión a 100x. Para asegurar que los 12 ejemplares seleccionados pertenecieran a la especie Lutzomyia longipalpis, se examinaron los 30 descriptores referenciados ut supra. Las imágenes de interés fueron fotografiadas usando una cámara digital AxioCam ERc5s fijada a un microscopio Zeiss Primo Star, y luego procesadas por el programa Axio Vision Rel 4.8.

\section{RESULTADOS}

De las observaciones microscópicas para la identificación rápida de Lutzomyia longipalpis surgió la conveniencia de utilizar cinco descriptores del aparato genital masculino, que en orden de importancia fueron:

1- setae diferenciadas del parámero (Figura 2).

2- relación entre longitud del parámero y lóbulo lateral 1:2 (Figura 3).

3- forma y apariencia del coxito o basistilo, ancho y robusto (Figura 4).

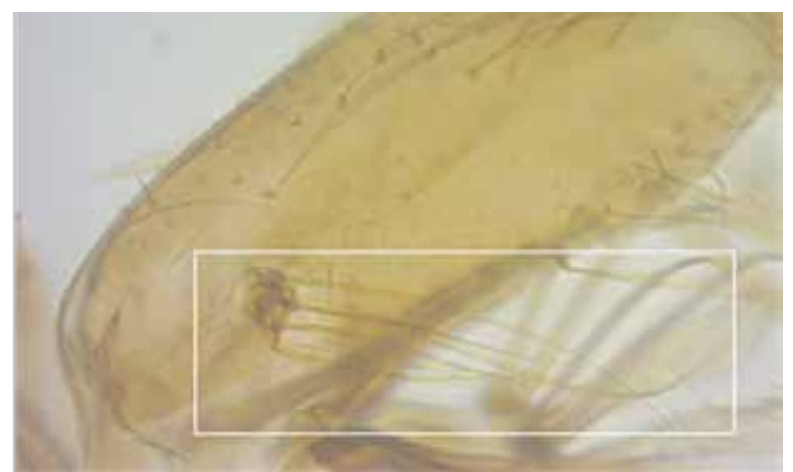

Figura 5. Características y ubicación del mechón de setae del coxito.

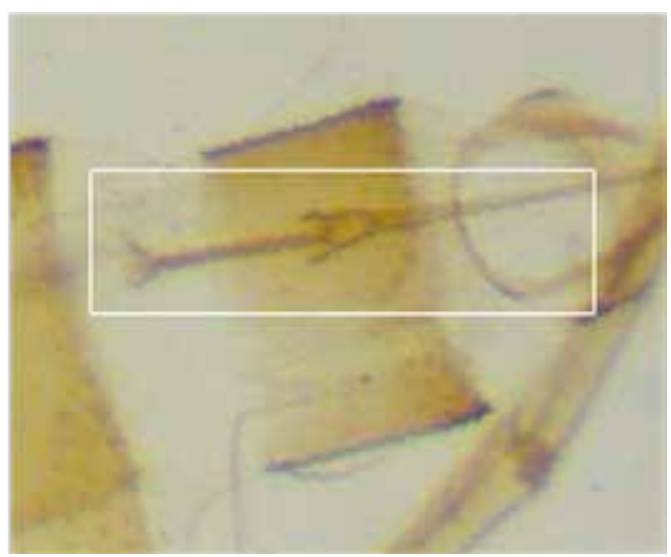

Figura 6. Bomba eyaculadora con pavilion estrecho y pistón largo. 
4- características y ubicación del mechón de setae del coxito: único, basal,

de 3 a 4 setae levemente separadas, implantadas en tubérculos (Figura 5).

5- bomba eyaculadora con pavilion estrecho y pistón largo (Figura 6).

Estos cinco descriptores se eligieron por ser los más relevantes y factibles de ser distinguidos por los operadores y/o técnicos encargados de las capturas, cumpliéndose así el objetivo planteado. Tales descriptores permiten identificar la especie en estudio de manera fidedigna, rápida y sencilla, diferenciándola de otras con características similares que también se encuentran en la zona,

\section{REFERENCIAS}

1. Cáceres AG, Villaseca P. 2002. Manual de procedimientos de identificación de vectores de leishmaniosis y enfermedad de Carrión. Serie de Normas Técnicas, Instituto Nacional de Salud (Lima, Perú) 36: 11-18.

2. Centro Nacional de Diagnóstico e Investigación de Enfermedades Endemo-epidémicas. 2011. Manual operativo para la comunidad. Publ. Red de Investigación de Leishmaniasis en Argentina (REDILA), Buenos Aires.

3. Dirección General de Salud Ambiental (DIGESA, Perú). 2002. Manual de campo para la vigilancia entomológica, Publ. Ministerio de Salud, Lima, p. 56-57.

4. Fernández CE, Tolezano JE, Galati EA. 2011. Chave de identificação ilustrada dos Phlebotominae (Diptera, Psychodidae) do estado de São Paulo, Brasil. Pap Avulsos Zool (São Paulo) 51: 83-100.

5. Laboratoire Informatique et Systématique (LIS, Paris). 2009. Base de datos de flebótomos de las Américas. On line: lis-upmc.snv.jussieu.fr/xper2/basesHtml/phle- botominae es/web//index.html.

6. Ministerio de la Protección Social, Instituto Nacional de Salud (Colombia). 2011. Gestión para la vigilancia entomológica y control de la transmisión de leishmaniasis. Medicina \& Laboratorio 17: 11-12.

7. Ministerio de Salud, Presidencia de la Nación (Argentina). 2010. Leishmaniasis visceral: guía para el equipo de salud, Publ. $\mathrm{N}^{\mathrm{0}} 5$, Dirección de Epidemiología, Buenos Aires, p. 5-7.

8. Programa de Estudio y Control de Enfermedades Tropicales (PECET). 2010. Manual de diagnóstico y control de la leishmaniasis en centroamérica, Publ. Universidad de Antioquia, Medellín (Colombia), p. 64-67.

9. Queiroz V, Paes P, Kleber L. 2003. Identificação de flebotomíneos de importância médica do Estado de Pernambuco. Publ. Universidade Federal de Pernambuco (Brasil). On lñine: www.ufpe.br/biolmol/Phlebotominaeon-line/ sumario.htm.

10. Salomon OD, Ramos LK, Quintana MG, Acardi SA, Santini MS, Schneider A. 2009. Distribución de vectores de Leishmaniasis visceral en la provincia de Corrientes. Medicina (Buenos Aires) 69: 625-630.

11. Salomon OD, Andrade Filho JD, Fernadez MS, Rosa JR, Szelag EA y Santini MS. 2010. Nuevos registros de Phlebotominae (Diptera: Psychodidae) para la Argentina. Rev. Soc. Entomol. Argent. 69: 3-4.

12. Torres Gutierrez C. 2009. Manual para el estudio e identificación de vectores de leishmaniasis. Documento guía del Curso Latinoamericano de Estudio e Identificación de Vectores de leishmaniasis (OMS-OPS-PECET), Medellín (Colombia), p. 52-54.

13. Vivero Gómez RJ. 2009. Guía fotográfica de flebotomíneos presentes en Colombia. Métodos de colecta. Publ. Programa de Estudio y Control de Enfermedades Tropicales (PECET), Universidad de Antioquia (Medellín, Colombia), p. 9.

14. Young DG. 1977. A biosystematic review of the bloodsucking psychodid flies of Colombia (Diptera: Phlebotominae and Sycoracinae). Ph. D. Thesis, University of Florida, p. 96-97 y 106-107. 